\title{
Recent data on Mediterranean diet, cardiovascular disease, cancer, diabetes and life expectancy
}

\author{
Ginter $\mathrm{E}^{1}$, Simko $\mathrm{V}^{2}$ \\ Institute of Preventive and Clinical Medicine, Bratislava, Slovakia. ginter.emil@mail.t-com.sk
}

\begin{abstract}
Benefits of dietary moderation when on a mediterranean diet type (MD) have been known for well over half a century. In the past, there has been a vigorous renewal of interest in preventive potential of MD. This review is unique, by focusing on the very recent confirmatory data on the MD, all published within the first half of 2014 . Benefits of MD in preventing and reducing cardiovascular disorders (CVD), known before, have been strongly confirmed. While there is little doubt regarding potential benefit of MD for obesity, diabetes type II, metabolic syndrome and fatty liver, critical evaluation has to be aimed at reported benefits of MD in such widely metabolic diverse disorders as cancer, pulmonary disease and cognition defects, including Alzheimer disease (Ref. 20). Text in PDF www.elis.sk.

Key words: mediterranean diet, cardiovascular diseases, hypertension, cancer, metabolic syndrome, diabetes, hepatic steatosis, life expectancy, oleocanthal, resveratrol, sirtuins, telomeres.
\end{abstract}

Mediterranean diet (MD) is characterized by abundant plant foods (fresh fruit, vegetables, breads, other forms of cereals, potatoes, beans, nuts, and seeds), olive oil as the principal source of fat, dairy products (principally cheese and yogurt), fish and poultry consumed in moderate amounts, red meat consumed in low amounts and wine.

\section{MD and vascular system}

Consistent evidence suggests that the promotion of the MD is an effective and feasible tool for the prevention of CVD (1). Although mixed nuts appear to be an important beneficial component in the MD, special emphasis is frequently attributed to extra virgin olive oil $(2,3)$.

Beneficial effect of MD in preventing CVD and thereby improving life expectancy (LE) were initially associated with the lowering of plasma cholesterol. Mechanism of MD effect is more complex. Not the least, consumption of MD reduces the blood pressure (4). Such effect of MD is possibly associated with lower risk of sudden cardiac death in postmenopausal women (5). Mediterranean diet rich in fat of vegetable origin may be a useful tool for the management of metabolic syndrome without a concern for weight gain due to its high fat content (6). In obese children and adolescents, MD significantly decreased the body mass index (BMI), fat mass, plasma glucose, total and LDL-cholesterol as well as triglycerides (7). MD resulted in an increased consumption of omega 9 fatty acids, zinc, vitamin E, selenium and decreased intake of saturated fatty acids.

${ }^{1}$ Institute of Preventive and Clinical Medicine, Bratislava, Slovakia, and ${ }^{2}$ State University of New York, Downstate Medical Centre at Brooklyn, USA

Address for correspondence: E. Ginter, RND, DSc, Racianska 17, SK83102 Bratislava, Slovakia.
Extensive metaanalysis confirmed the prominent effect of MD on outcomes of endothelial function and inflammation (8). MD resulted in a significant increase in flow mediated dilatation and adiponectin, while pro-inflammatory $\mathrm{C}$ reactive protein, interleukin-6 and intracellular adhesion molecule- 1 were significantly decreased. Extra virgin olive oil incorporated into MD, blunts post-prandial oxidative stress via down-regulation of nicotinamide adenine dinucleotide phosphate-oxidase (NOX2) (9).

Another protective factor in MD may be the magnesium. The relation between dietary magnesium intake and CVD mortality was evaluated in several prospective studies. MD contains a generous amount of Mg. Magnesium intake from MD was inversely associated with cardiovascular, cancer and all-cause mortality (10).

\section{MD and type 2 diabetes}

Diabetes is an important metabolic disorder contributing to CVD. An extensive metaanalysis including 136,846 participants indicated that a higher adherence to MD was associated with 23 $\%$ reduced risk of developing type 2 diabetes (11). In patients with newly diagnosed type 2 diabetes, consumption of MD resulted in a greater reduction of $\mathrm{HbA1c}$ levels, higher rate of diabetes remission, and delayed need for diabetes medication, compared to a less specific low-fat diet (12). MD enriched with extra-virgin olive oil but without energy restrictions reduced diabetes risk among persons with a high cardiovascular risk (13).

\section{MD and cancer}

Past reports regarding a potential preventive effect of MD in cancerogenesis were considered doubtful. New light on this con- 
troversy was shed with an extensive metaanalysis that was based on twenty-one cohort studies including 1,368,736 subjects and 12 case-control studies, with 62,725 subjects. These populations met the review objectives and were enclosed for meta-analyses (14). A high adherence to MD was associated with a significant reduction in the risk of overall cancer mortality (10\%), colorectal cancer (14\%) and prostate cancer. Nonsignificant changes were for breast cancer, gastric cancer and pancreatic cancer. Another study provided the evidence of a beneficial role of MD on cancer of the oral cavity and pharyngeal malignancy (15).

The biological mechanisms for cancer prevention associated with the Mediterranean diet have been related to the favourable effect of a balanced ratio of omega 6 and omega 3 essential fatty acids, as well as the high amount of fibre, antioxidants and polyphenols found in fruit, vegetables, olive oil and wine.

Benefits of MD supposedly also extend, in addition to cancer, to other non communicable diseases, to the Parkinson and Alzheimer disease. Several components of the MD have demonstrated benefits in controlling the pathophysiological mechanisms involved in preventing the nonalcoholic fatty liver disease (16).

Recently, positive effects of MD were attributed to the presence of polyphenols and their derivatives (e.g. oleocanthal) that by exerting an anti-inflammatory and anti-oxidative effect can be involved in the reduction of age-related pathologies, such as cardiovascular, neoplastic, metabolic and neurodegenerative diseases (17). The scientific explanation of such beneficial effects was limited to the reduction of the oxidative stress by compounds present in the MD. A new class of proteins called sirtuins have gained the attention because of their antiageing effects, their ability to protect from cardiovascular, metabolic, neurodegenerative diseases, cancer and to extend the lifespan. Resveratrol, a polyphenol present in grapes, nuts and berries has been shown to activate sirtuins and such activation is able to explain some beneficial effects of the MD (18).

\section{MD, longevity and telomeres}

In 1970, The Seven Countries Study found that Cretan men had an exceptionally high LE and low death rates from heart disease, despite moderate to high intake of fat. Most recent data on LE in the Mediterranean countries indicate male LE to be close to 80 years (Italy and Cyprus), female LE (Spain, France and Italy) to be close to 85 years, being some of the highest LE in Europe.

Postulated beneficial effect of MD is related to prevention of shortening of telomeres. Telomeres are nucleoprotein structures that protect the ends of eukaryocyte chromosomes. In depth research on MD supports a novel role of MD in promoting health span, suggesting that telomere maintenance is the major determinant of health among the elderly (17). Shorter telomere length is associated with several age-related human disorders and subsequent shorter LE. Telomere length is a better indicator of LE than chronological age. Telomere length and kinetic predict mortality. An increase in telomere length was noted after a five year MD intervention (18).

\section{Conclusion}

There is enough evidence that a diet rich in plant fibre, phytosterols, fatty acids derived from fish and plant oil, is beneficial. Population residing in the Mediterranean territory outperforms other Europeans in longevity and lower incidence of cardiovascular disorders. Benefits of a Mediterranean diet (MD) are well supported by solid scientific evidence based on outcome of proinflammatory factors, as well as very credible data on cell biological components, nucleoprotein telomeres and anti-ageing sirtuins. Still, critical appraisal is needed for overly enthusiastic reports coming out of the coffee grinder fed by metaanalyses, based on enormous number of observed individuals. Large epidemiological studies are subject to potential bias induced by inclusion of hard to control confusing variables. MD is not a strictly defined formula and it varies between communities and individuals. A selection bias in large dietary studies may be induced by the extent of health consciousness in dieting individuals. Non dietary factors present in the Mediterranean littoral are usually not considered: level of exercise, vitamin D saturation from sun exposure, role of alcohol intake and smoking. There is a natural inclination, not always productive, for enthusiastic acceptance of one of the most natural component of alternative medicine, the food. Cancer, pulmonary disease and cognitive disorders, including Parkinson and Alzheimer, represent widely divergent pathophysiological processes. Best research and multibillion investments have been trying to decipher these troubling maladies. It appears too fortuitous for a diet to offer a universally applicable miracle answer.

\section{References}

1. Martinez-Gonzalez MA, Bes-Rastrollo M. Dietary patterns, Mediterranean diet, and cardiovascular disease. Curr Opin Lipidol 2014; 25 (1): 20-26.

2. Whayne TF Jr. Ischemic heart disease and the Mediterranean diet. Curr Cardiol Rep 2014; 16 (6): 491. doi: 10.1007/s11886-014-0491-6.

3. Guasch-Ferré M, Hu FB, Martínez-González MA et al. Olive oil intake and risk of cardiovascular disease and mortality in the PREDIMED Study. BMC Med 2014; 12: 78. doi: 10.1186/1741-7015-12-78.

4. Doménech M, Roman P, Lapetra J et al. Mediterranean diet reduces 24-hour ambulatory blood pressure, blood glucose, and lipids: one-year randomized, clinical trial. Hypertension 2014; 64 (1): 69-76.

5. Bertoia ML, Triche EW, Michaud DS et al. Mediterranean and Dietary Approaches to stop hypertension dietary patterns and risk of sudden cardiac death in postmenopausal women. Am J Clin Nutr 2014; 99 (2): 344-351.

6. PREDIMED Study Investigators. Effects of 1-year intervention with a mediterranean diet on plasma Fatty acid composition and metabolic syndrome in a population at high cardiovascular risk. PLoS One 2014; 9 (3): e85202.

7. Velázquez-López L, Santiago-Díaz G, Nava-Hernández J et al. Mediterranean-style diet reduces metabolic syndrome components in obese children and adolescents with obesity. BMC Pediatr 2014; 14 (1): 175. doi: 10.1186/1471-2431-14-175.

8. Schwingshackl L, Hoffmann G. Mediterranean dietary pattern, inflammation and endothelial function: A systematic review and meta-analysis 


\section{6-348}

of intervention trials. Nutr Metab Cardiovasc Dis 2014 Apr 2. pii: S09394753(14)00109-4.

9. Carnevale R, Pignatelli P, Nocella C et al. Extra virgin olive oil blunt post-prandial oxidative stress via NOX2 down-regulation. Atherosclerosis 2014; 235 (2): 649-658.

10. Guasch-Ferré M, Bulló M, Estruch R et al. Dietary magnesium intake is inversely associated with mortality in adults at high cardiovascular disease risk. J Nutr 2014; 144 (1): 55-60.

11. Koloverou E, Esposito K, Giugliano D, Panagiotakos D. The effect of Mediterranean diet on the development of type 2 diabetes mellitus: a meta-analysis of 10 prospective studies and 136,846 participants. Metabolism 2014; 63 (7): 903-911.

12. Esposito K, Maiorino MI, Petrizzo M et al. The effects of a Mediterranean Diet on the need for diabetes drugs and remission of bewly diagnosed type 2 diabetes: Follow-up of a randomized trial. Diabetes Care 2014; 37 (7): 1824-1830.

13. Salas-Salvadó J, Bulló M, Estruch R et al. Prevention of diabetes with Mediterranean diets: a subgroup analysis of a randomized trial. Ann Intern Med 2014; 160 (1): 1-10.

14. Schwingshackl L, Hoffmann G. Adherence to Mediterranean diet and risk of cancer: A systematic review and meta-analysis of observational studies. Int J Cancer 2014 Mar 6. doi: 10.1002/ijc.28824.
15. Filomeno M, Bosetti C, Garavello W et al. The role of a Mediterranean diet on the risk of oral and pharyngeal cancer. Br J Cancer 2014 Jun 17. doi: 10.1038/bjc.2014.329.

16. Velasco N, Contreras A, Grassi B. The mediterranean diet, hepatic steatosis and nonalcoholic fatty liver disease. Curr Opin Clin Nutr Metab Care 2014 Jul 11.

17. Parkinson L, Keast R. Oleocanthal, a phenolic derived from virgin olive oil: a review of the beneficial effects on inflammatory disease. Int $\mathrm{J}$ Mol Sci 2014; 15 (7): 12323-12334.

18. Russo MA, Sansone L, Polletta L et al. Sirtuins and Resveratrolderived compounds. A model for understanding the beneficial effects of the Mediterranean Diet. Endocr Metab Immune Disord Drug Targets. 2014 Jul 8.

19. Boccardi V, Esposito A, Rizzo MR et al. Mediterranean diet, telomere maintenance and health status among elderly. PLoS One 2013; 8 (4): e62781. doi: 10.1371.

20. García-Calzón S, Gea A, Razquin C et al. Longitudinal association of telomere length and obesity indices in an intervention study with a Mediterranean diet: the PREDIMED-NAVARRA trial. Int J Obes (Lond) 2014; 38 (2): 177-182.

Received September 1, 2014. Accepted October 1, 2014. 